ORIGINAL RESEARCH

\title{
Field Ultrasound Evaluation of Central Volume Status and Acute Mountain Sickness
}

\author{
Justin T. Pitman, MD; Ghan B. Thapa, MD; N. Stuart Harris, MD, MFA \\ From the Department of Emergency Medicine, Massachusetts General Hospital, Boston, MA (Drs Pitman and Harris); and the Mountain Medicine \\ Society of Nepal, Maharajgunj, Kathmandu, Bagmati, Nepal (Dr Thapa).
}

\begin{abstract}
Objective.-To investigate whether ultrasonography can be used for field volume status assessment and to determine whether a detectable difference in intravascular volume exists in individuals with acute mountain sickness (AMS) compared with those without.

Methods.-Study was performed at the Himalayan Rescue Association Clinic in Manang, Nepal, located on the Annapurna trekking circuit at an altitude of $3519 \mathrm{~m}$ (11545 feet). A convenience sample was taken from individuals trekking over 5 to 8 days from $760 \mathrm{~m}$ (2490 feet) to $3519 \mathrm{~m}$ (11,545 feet), comparing asymptomatic trekkers vs those who experienced AMS. Subjects were evaluated for AMS based on the Lake Louise AMS Questionnaire (LLS $\geq 3$ indicates AMS). After medical screening examination, both groups (control, $\mathrm{n}=51$; AMS, $\mathrm{n}=18$ ) underwent ultrasonography to obtain measurements of inferior vena cava collapsibility index (IVC CI) and left ventricular outflow tract velocity-time integral (LVOT VTI) before and after a passive leg raise (PLR) maneuver.

Results.-There was no statistically significant difference between groups regarding change in heart rate before and after PLR, or IVC CI; however, there was a statistically significant greater increase in LVOT VTI after PLR maneuver in control group subjects compared with those with AMS (18.96\% control vs $11.71 \%$ AMS; $P<.01)$.

Conclusions.- Ultrasonography is a useful tool in the assessment of intravascular volume at altitude. In this sample, we found ultrasonographic evidence that subjects with AMS have a higher intravascular volume than asymptomatic individuals. These data support the hypothesis that individuals with AMS have decreased altitude-related diuresis compared with asymptomatic individuals.
\end{abstract}

Key words: acute mountain sickness, intravascular volume

\section{Introduction}

Acute mountain sickness (AMS) is a common clinical syndrome that occurs at altitudes above $2500 \mathrm{~m}(8000$ feet). The clinical symptoms of AMS include headache and one of the following: gastrointestinal upset, fatigue or weakness, dizziness, or sleep disturbance. ${ }^{1}$ Severity and incidence of these conditions depend on the rate of ascent, elevation obtained (especially sleeping altitude), duration of altitude exposure, physical exertion, and inherent genetic susceptibility. ${ }^{2-4}$ On acute exposure to $2500 \mathrm{~m}$ (8000 feet), approximately $25 \%$ of travelers experience symptoms of AMS, and $1 \%$ to $2 \%$ of people exhibit the life-threatening conditions of high altitude

Corresponding author: Justin T. Pitman, MD, Department of Emergency Medicine, Massachusetts General Hospital, 55 Fruit Street, Zero Emerson, Suite 3B, Boston, MA 02114 (e-mail: jpitman@mgh. harvard.edu) cerebral or pulmonary edema (HACE, HAPE, respectively)..$^{5-8}$ The underlying pathophysiology of AMS remains poorly defined, but some data support the role of increased fluid retention, which may contribute to cerebral overperfusion, endothelial leakage, and eventual subclinical cerebral edema. ${ }^{3}$

Although the process of acclimatization to altitude is multifactorial and complex, data suggest individuals who experience AMS undergo a blunted bicarbonate $\left[\mathrm{HCO}^{-}\right]$diuretic response on exposure to a high altitude environment., ${ }^{9,10}$ Various mechanisms have been proposed for this, including blunted hypoxic ventilatory response ${ }^{6,9,11,12}$ and diminished renal perfusion secondary to sympathetic overstimulation. ${ }^{13,14}$ It has been demonstrated that individuals with AMS have a neutral or net gain of intravascular volume compared with a net loss of intravascular volume in individuals who remain asymptomatic. $^{10,11,14}$ 
Numerous studies have been published using bedside ultrasonography (US) to assess intravascular volume status in patients in emergency department and intensive care unit settings. ${ }^{15-22}$ Two of the described techniques are of particular interest for volume status assessment: the inferior vena cava collapsibility index (IVC CI), and left ventricular outflow tract velocity-time integral (LVOT VTI) before and after a passive leg raise (PLR) maneuver. The IVC CI measures the change in the IVC diameter during the patient's respiratory cycle. Negative intrathoracic pressure generated during inspiration improves cardiac venous return, which leads to a collapse of the thin-walled IVC and increases right ventricular preload and pulmonary venous capacitance. The degree of IVC collapse with inspiration varies with the intravascular volume state of the individual: hypovolemic individuals have large degrees of collapse, euvolemic subjects have moderate collapse, and hypervolemic subjects have minimal to no collapse. The degree of IVC collapse can be directly imaged with US and measured. A collapse of the IVC by $50 \%$ or more is suggestive of a low intravascular volume status and a fluid responsive state (1-10\% notes minimal collapse; $100 \%$ notes complete collapse [marked hypovolemia]). ${ }^{18,20,21}$

The LVOT VTI technique provides information regarding left ventricular ejection velocity. As a pulsatile system, blood flow is not constant, but rather undergoes rapid acceleration and deceleration during ventricular contraction. By using a Doppler-enabled US, one can measure these changes in velocity and generate an ejection velocity waveform. By performing an integral calculation of the ejection velocity waveform as measured during a single cardiac cycle, the calculated number is expressed as the LVOT VTI. From this information, further physiologic parameters can then be derived as well. LVOT VTI multiplied by the LVOT area can assess beat-to-beat stroke volume, and, when multiplied by heart rate, cardiac output. LVOT VTI, when taken alone, is highly variable among individuals and is difficult to compare. However, dynamic changes can be measured and standardized. Performing a PLR involves transitioning subject from a semirecumbent position (with legs on bed in front of subject) to a supine position with legs raised to $45^{\circ}$. Raising the legs results in lower extremity pooled venous blood to be rapidly returned to the central venous system, which is mechanistically analogous to an intravascular fluid bolus (Figure 1). ${ }^{15,23-25}$ Based on the Frank-Starling law of the heart, as venous return improves, ejection velocity, stroke volume, and cardiac output also improve. The largest gains in ejection velocity and stroke volume occur when preload is low (hypovolemia) and volume is added to the system. However, as preload is increased,

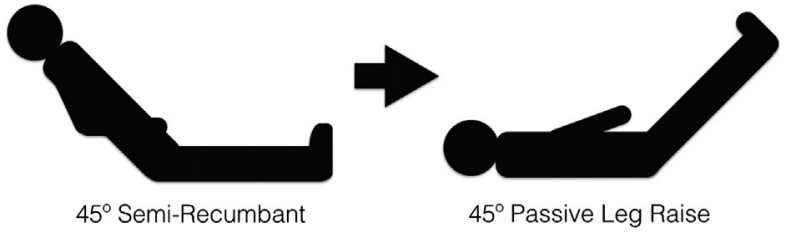

Figure 1. Left ventricular outflow tract velocity-time integral measurements are first taken while subject is in a $45^{\circ}$ semirecumbent position. The subject is then moved into a supine position and legs are passively elevated to $45^{\circ}$, rapidly returning pooled venous blood to the central venous circulation and subsequently increasing left ventricular ejection velocity and stroke volume. A $>12 \%$ increase in the left ventricular outflow tract velocity-time integral with passive leg raise gives evidence of a hypovolemic volume state. These individuals are "fluid responsive."

output gains are reduced, and ultimately reversed as the ventricles are stretched beyond limits of maximal contractility. Previously reported literature in both emergency department and intensive care unit settings have reported changes in LVOT VTI before and after PLR based on volume status. Individuals who improve LVOT VTI by $12 \%$ or more after a PLR maneuver have been shown prospectively to improve cardiac output and endorgan perfusion after an intravenous fluid bolus, and were thus hypovolemic. ${ }^{16,23,24,26}$ Although the images are technically more difficult than IVC CI to obtain, Royse et $\mathrm{al}^{27}$ demonstrated that after only 20 studies, even novice US users obtain accurate and reproducible data.

The aims of this study are to determine whether US assessment of subject volume status using IVC CI and LVOT VTI before and after a PLR maneuver is feasible in a wilderness setting and to demonstrate whether these techniques are capable of detecting intravascular volume differences between an AMS cohort and a healthy control group.

\section{Methods}

\section{SUBJECT SELECTION}

Subject recruitment took place through the Manang Branch of the Himalayan Rescue Association at an altitude of $3519 \mathrm{~m}$ (11545 feet). All of the subjects began the Annapurna Circuit trek in Besisahar at $760 \mathrm{~m}$ (2490 feet) and after 5 to 8 days of trekking, reached Manang, an altitude gain of 2759 m (9055 feet). Recruitment posters were placed at mandatory checkpoints and popular tea houses throughout the trek. Subjects, both with and without symptoms of AMS, volunteered either spontaneously or after attending our daily altitude sickness lecture, or were recruited through our clinic. Enrollment criteria included 1) ascent to Manang, Nepal, recruitment center 3519 m (11545 feet) between September 24 and December 2, 2013, 2) 
altitude exposure above $2500 \mathrm{~m}$ (8000 feet) for less than 7 days, 3) inclusion in the AMS cohort required presence of headache and at least one other symptom required for the diagnosis of AMS (including nausea, vomiting, fatigue, weakness, dizziness, lightheadedness, or poor sleeping) as defined by the Lake Louse AMS questionnaire. ${ }^{1}$ Subjects not meeting Lake Louise AMS criteria were assigned to the asymptomatic control group. Exclusion criteria included 1) age younger than 18 years or older than 65 years; 2) more than 7 days at altitudes above $2500 \mathrm{~m}$ (8000 feet); 3) evidence of severe high altitude illness (eg, HAPE as evidenced by dyspnea at rest, or HACE as evidenced by altered mental status or ataxia); 4) known or suspected pregnancy; and 5) history of cardiovascular disease, congestive heart failure, chronic obstructive pulmonary disease, pulmonary hypertension, interstitial lung disease, or other chronic cardiac or pulmonary disorder. Study protocol has been submitted, reviewed, and approved by the Partners/ Massachusetts General Hospital Institution Review Board and the Nepal Health Research Council.

Eighty-six subjects were evaluated for possible enrollment in this study. Ten subjects were excluded after screening examination revealed previous altitude exposure within the past week, 4 subjects declined participation in the study citing symptoms of AMS, 2 subjects were excluded based on the clinical diagnosis of HACE, and 1 subject was excluded based on clinical diagnosis of HAPE. A total of 69 subjects were enrolled between September 24 and December 2, 2013. Fifty-one met criteria for the control group and 18 subjects met AMS group criteria. Subjects were assigned numbers in order of presentation, and information was recorded anonymously. Epidemiologic data regarding age, sex, country of origin, trekking day number, past medical history, and whether the individual had used acetazolamide was recorded. Given highly variable compliance and prescription patterns, acetazolamide use was recorded in binary fashion: yes indicates an individual had used this medication at least once during ascent. Eligible subjects underwent a screening medical examination, including heart rate, blood pressure, respiratory rate, and pulse oximetry. Values were obtained while the patient was in a seated position. Records were kept in a locked room or on the investigator's person during travel. Ultrasonographic images were labeled with corresponding subject number for identification purposes. To every extent possible, blinded review of US images were attempted. Blinded IVC CI measurements were performed offline by a single reviewer. However, given technological limitations, LVOT VTI measurements required calculation in real time, and subsequently were not subjected to blind image review. Both IVC CI and LVOT VTI screen shots were captured after a suitable high-quality image was obtained, and repeated for a total of 3 times. Images suitable for measurement were obtained in all 69 enrolled patients. IVC CI images from a single control group subject were unable to be located during review and thus are not included in the statistical analysis.

\section{ULTRASONOGRAPHY TECHNIQUES}

All ultrasound images were gathered using the SonoSite M-Turbo (SonoSite Inc, Bothell, WA) (weight, $3.04 \mathrm{~kg}$ ) combined with cardiac phase array probe ( $\mathrm{P} 21 \mathrm{x}$ Transducer, 5-1 MHz, 17-mm foot print, scan depth $35 \mathrm{~cm}$ ). Equipment was provided by SonoSite for research purposes and returned after completion of study. The author's (JTP) experience with ultrasound included a 4year emergency medicine residency with credentialing in emergency ultrasound, and 5 dedicated scanning periods with emergency medicine ultrasound faculty to practice both IVC CI and LVOT VTI techniques.

\section{INFERIOR VENA CAVA COLLAPSIBILITY INDEX}

Subjects were placed on a stretcher in a semirecumbent position with their chest angled at $45^{\circ}$. The ultrasound probe was placed adjacent to the subject's xiphoid process overlying the hepatic segment of the IVC and visualized in the longitudinal plane. Longitudinal 2dimensional (2D) cine loops spanning at least one respiratory cycle were obtained. IVC maximum diameter was measured at a point $2 \mathrm{~cm}$ from right atrium at both end expiration (IVCe) and end inspiration (IVCi; Figure 2). Measurements were performed 3 times to ensure the plane of the US beam was at maximum diameter and saved for offline statistical analysis. The collapsibility index of the IVC was calculated as IVC $\mathrm{CI}=[(\mathrm{IVCe}-\mathrm{IVCi}) / \mathrm{IVCe}] \times 100$.

\section{LEFT VENTRICULAR OUTFLOW TRACT VELOCITY-TIME INTEGRAL}

Subjects remained in semirecumbent position. The US probe was placed adjacent to the left side of the subject's sternum to obtain the parasternal long view and LVOT diameter was measured at the level of the aortic annulus. The probe was then moved several centimeters below the subject's left nipple to obtain a periapical 5 view. LVOT VTI measurements were taken by positioning the pulse wave Doppler probe within $0.5 \mathrm{~cm}$ of the aortic valve on the ventricular side, and the subsequent waveform was traced to obtain the VTI (Figure 3). Three separate measurements were taken to ensure measurement reliability. Stroke volume (SV) calculations were obtained by $\mathrm{SV}=$ LVOT VTI $\times(\text { LVOT diameter })^{2} \times 0.78540$. 

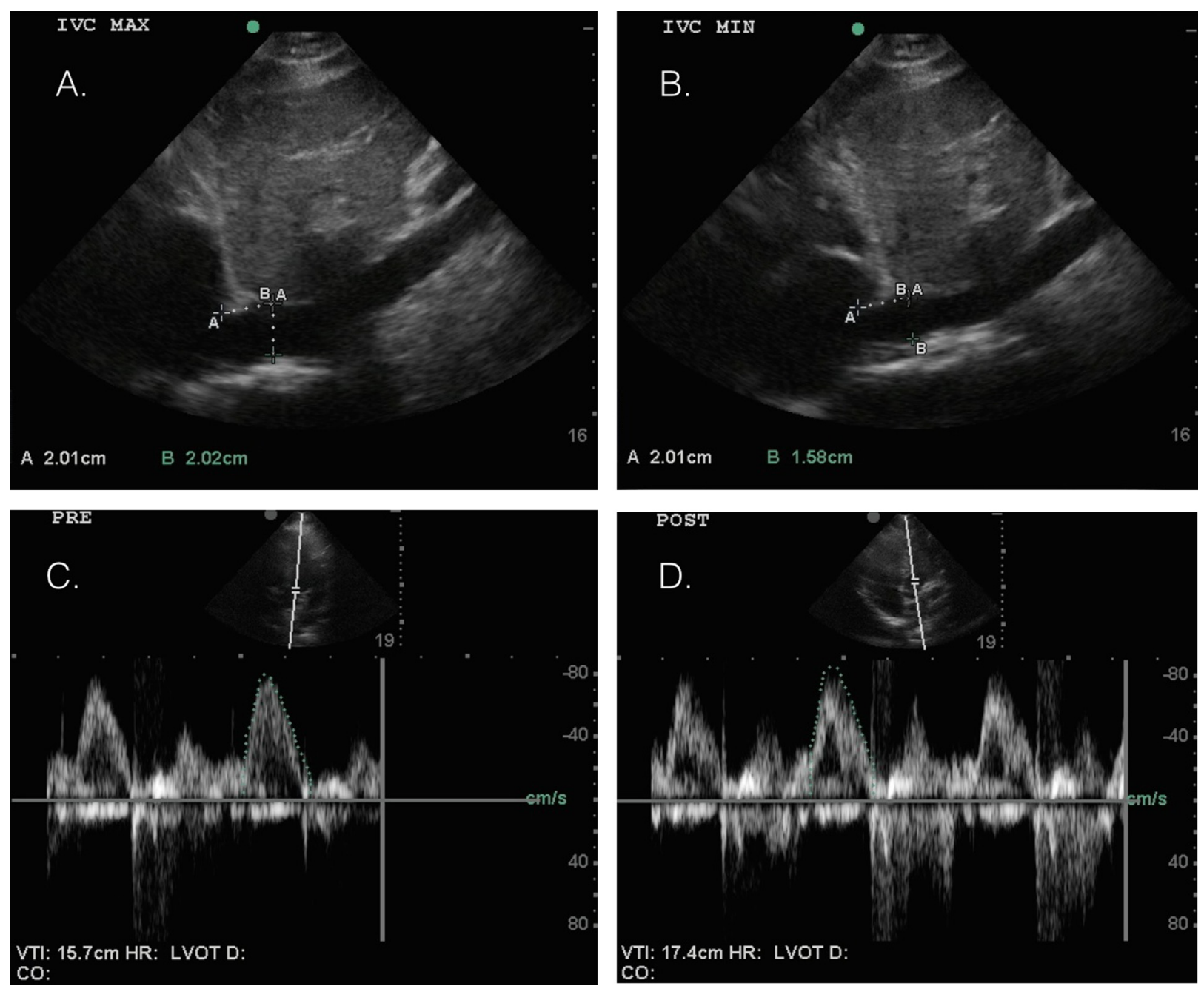

Figure 2. Inferior vena cava collapsibility index (IVC CI) is determined by ultrasonographic view of the IVC in the sagittal plane at endexpiration (A) and at end-inspiration (B). As the subject inspires, negative intrathoracic pressure enhances cardiac venous return and decreases the diameter of the thin-walled IVC. To obtain this measurement, the probe is held in the sagittal plane, and a cine clip is recorded during the subject's respiratory cycle. The IVC diameter is measured at a point $2 \mathrm{~cm}$ caudally from the right atrium. IVC CI is calculated using (IVCe - IVCi) / IVCe. A collapse of $>50 \%$ is suggestive of a low intravascular volume status. Left ventricular outflow tract velocity-time integral (LVOT VTI) waveforms obtained with subject $45^{\circ}$ semirecumbent (C) and supine with $45^{\circ}$ passive leg raise (D) using an apical 5 view with Doppler probe placed at the level of the left ventricular outflow tract. The integral of the waveform is directly proportional to left ventricular ejection volume. When the subject transitions from semirecumbent to passive leg raise position, the increased venous return causes an increase in the LVOT VTI. An increase $>12 \%$ suggests low intravascular volume status.

Cardiac output $(\mathrm{CO})$ calculations were obtained by $\mathrm{SV} \times$ $\mathrm{HR}$, where $\mathrm{HR}$ is the heart rate. After measurements were taken in a semirecumbent position, subjects were then moved to a supine position and legs were passively elevated to $45^{\circ}$. After a period of 2 to 3 minutes, allowing time for central venous volume to equilibrate, both heart rate and LVOT VTI values were measured again. Change in heart rate $(\Delta H R)$ before and after PLR was calculated using the following equation: $\Delta \mathrm{HR}=$ post-HR - pre-HR. Percent increase in LVOT VTI (\%Inc LVOT VTI) after PLR maneuver was calculated using the formula \%Inc LVOT VTI $=$ (post-LVOT VTI - preLVOT)/(post-LVOT VTI). Given that the LVOT diameter is fixed, the percent increase in cardiac output (\% Inc CO) after PLR testing can be measured by \%Inc CO $=([$ PostLVOT VTI $\times$ Post-HR] $-[$ Pre-LVOT VTI $\times$ Pre-HR] $) /$ (Post-LVOT VTI $\times$ Post-HR).

\section{STATISTICAL ANALYSIS}

Data for $\Delta \mathrm{HR}$, IVC CI, and \%Inc CO in subjects with and without symptoms of AMS were compared using 

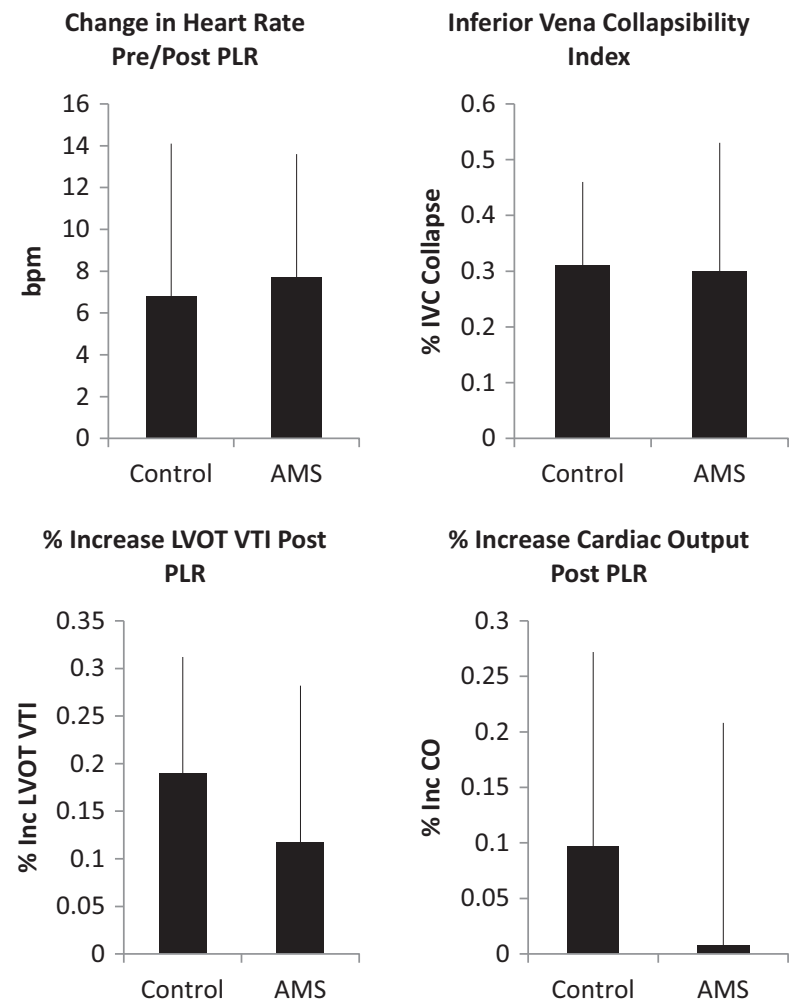

Figure 3. Change in heart rate ( 6.8 beats/min control vs 7.7 beats/min acute mountain sickness [AMS]), inferior vena cava (IVC) collapsibility index (30.5\% control vs $29.6 \%$ AMS), and cardiac output increase (Inc CO: 9.7\% control vs 0.8\% AMS) did not meet statistical significance. However, the left ventricular outflow tract velocity-time integral (LVOT VTI) increased after passive leg raise (18\% control vs $11 \%$ AMS; $P=.0088)$.

the $\chi^{2}$ test. The \%Inc LVOT VTI comparison between groups was calculated with a repeated-measures analysis of variance. All data were analyzed using Statistical Analysis Systems 9.3 (SAS Institute, Cary, NC). All data are reported as mean $\pm \mathrm{SD}$, with the significance level set at $P<.05$.

\section{Results}

Tables 1 and 2 summarize the patient demographic data. The Lake Louise AMS score in the control group averaged $0.86 \pm 0.83$, whereas the AMS score in the AMS group averaged $5.06 \pm 2.18$. Acetazolamide was used by $29.4 \%$ of the control subjects compared with $55.6 \%$ of the subjects with AMS. No significant difference was found in $\triangle H R$ before and after a PLR maneuver between control and AMS subjects $(6.8 \pm$ 7.3 beats $/ \mathrm{min}$ vs $7.7 \pm 5.9$ beats $/ \mathrm{min} ; P=.59$ ). Ultrasonographic evaluation of IVC CI did not demonstrate any statistically significant difference $(30.5 \% \pm 15 \%$ control vs $29.6 \% \pm 22.8 \%$ AMS; $P=.87$ ). The percent increase in LVOT VTI before and after PLR, however, using repeated-measures analysis of variance did demonstrate statistical significance $(18.96 \% \pm 12.5 \%$ control vs $11.71 \% \pm 16.48 \%$ AMS; $P=.0088$ ), but $\%$ Inc CO using before and after PLR HR values did not $(9.7 \% \pm$ $17.5 \%$ control vs $0.8 \% \pm 20 \% ; P=.1$; Figures 3,4 ).

\section{Discussion}

The use of ultrasonography in the wilderness setting has rapidly been adopted for field use given its portability and wide range of both clinical and research applications. ${ }^{28-31}$ Here, we demonstrate the feasibility of volume status measurement by ultrasound in a rugged, high altitude environment. To our knowledge, this study represents the first application of this technique to the altitude-exposed patient. Based on our results, we were able to detect a statistically significant difference in intravascular volume in subjects with and without AMS via the surrogate marker of LVOT VTI measurements combined with a PLR maneuver. Given marked individual variability, this technique likely will have limited utility for assessing a single patient at a single time. Rather, this technique will be more suited for detecting intravascular volume change in a single subject by serial measurements. In this study, change in HR before and after PLR and IVC CI do not appear to be useful markers of intravascular volume status in the setting of AMS.

Field studies such as Hackett et $\mathrm{al}^{9}$ and simulated altitude chamber studies such as Loeppky et $\mathrm{al}^{10}$ and

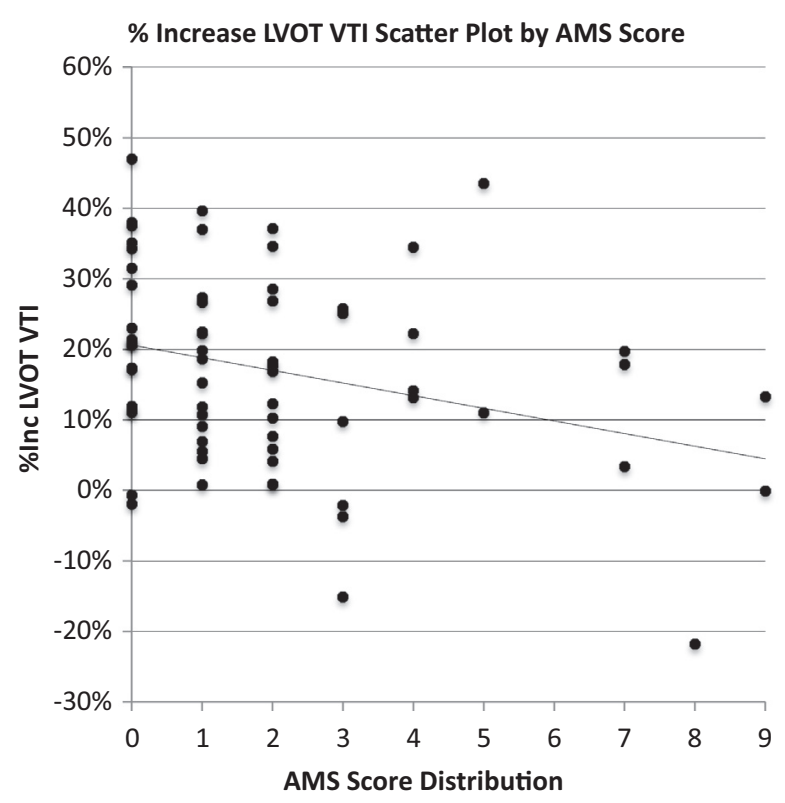

Figure 4. Scatterplot of Lake Louise acute mountain sickness (AMS) score vs $\%$ increase in left ventricular outflow tract velocity-time integral (LVOT VTI) after passive leg raise maneuver. 
Table 1. Clinical characteristics of acute mountain sickness and control subjects at presentation

\begin{tabular}{|c|c|c|c|}
\hline & Control & $A M S$ & $P$ value \\
\hline Total patients & 51 & 18 & \\
\hline \multicolumn{4}{|l|}{ Age, y } \\
\hline Mean & $37 \pm 10.8$ & $38.2 \pm 11.7$ & .71 \\
\hline Range & $23-62$ & $23-56$ & \\
\hline Male sex, n $(\%)$ & $24(47.1 \%)$ & $7(44.9 \%)$ & \\
\hline Trekking day & $5.96 \pm 1.49$ & $6.41 \pm 1.33$ & \\
\hline \multicolumn{4}{|l|}{ Acetazolamide, n (\%) } \\
\hline No & $35(68.6 \%)$ & $10(55.6 \%)$ & \\
\hline Yes & $15(29.4 \%)$ & $8(44.4 \%)$ & \\
\hline AMS score & $0.86 \pm 0.83$ & $5.06 \pm 2.18$ & \\
\hline Range & $0-2$ & $3-9$ & \\
\hline \multicolumn{4}{|l|}{ Heart rate, beats/min } \\
\hline Semirecumbent & $73.3 \pm 11.6$ & $71.6 \pm 12.1$ & .61 \\
\hline Supine with leg raise & $66.2 \pm 11.8$ & $63.8 \pm 10.2$ & .43 \\
\hline Delta HR & $6.8 \pm 7.3$ & $7.7 \pm 5.9$ & .59 \\
\hline $\mathrm{O}_{2}$ Sat, $\%$ & $93 \pm 3$ & $93 \pm 3$ & .9 \\
\hline \multicolumn{4}{|l|}{ Blood pressure } \\
\hline $\mathrm{SBP}, \mathrm{mm} \mathrm{Hg}$ & $120.2 \pm 12.3$ & $123.3 \pm 18.5$ & .52 \\
\hline $\mathrm{DBP}, \mathrm{mm} \mathrm{Hg}$ & $76 \pm 9.6$ & $80 \pm 11.6$ & .39 \\
\hline \multicolumn{4}{|l|}{ IVC } \\
\hline Maximum, $\mathrm{cm}$ & $1.94 \pm 0.39$ & $1.97 \pm 0.3$ & .75 \\
\hline Minimum, $\mathrm{cm}$ & $1.35 \pm 0.39$ & $1.38 \pm 0.49$ & .81 \\
\hline Collapsibility index & $30.5 \% \pm 15$ & $29.2 \% \pm 22.8$ & .87 \\
\hline \multicolumn{4}{|l|}{ LVOT } \\
\hline Diameter, cm & $1.66 \pm 0.18$ & $1.64 \pm 0.26$ & .69 \\
\hline VTI (pre-PLR), cm & $15.44 \pm 2.74$ & $16.16 \pm 3.95$ & .48 \\
\hline VTI (post-PLR), cm & $19.17 \pm 2.77$ & $18.31 \pm 2.49$ & .23 \\
\hline \% increase VTI with PLR & $18.96 \% \pm 12.25$ & $11.71 \% \pm 16.48$ & $.0088^{a}$ \\
\hline \multicolumn{4}{|l|}{ Stroke volume (SV) } \\
\hline $\mathrm{SV}$ (pre-PLR), mL & $34.1 \pm 9.7$ & $33.4 \pm 8.6$ & .78 \\
\hline SV (post-PLR), mL & $42.4 \pm 11.6$ & $38.6 \pm 10.4$ & .21 \\
\hline$\%$ increase $\mathrm{SV}$ & $19 \% \pm 12.2$ & $11.7 \% \pm 16.5$ & .1 \\
\hline \multicolumn{4}{|l|}{ Cardiac output $(\mathrm{CO})(\mathrm{L} / \mathrm{min})$} \\
\hline $\mathrm{CO}$ (pre-PLR) L/min & $2.47 \pm 0.82$ & $2.38 \pm 0.75$ & .65 \\
\hline $\mathrm{CO}$ (post-PLR) L/min & $2.8 \pm 0.93$ & $2.44 \pm 0.70$ & .094 \\
\hline$\%$ increase $\mathrm{CO}$ with PLR & $9.7 \% \pm 17.5$ & $0.8 \% \pm 20$ & .11 \\
\hline
\end{tabular}

All values expressed as mean $\pm \mathrm{SD}$.

${ }^{a} P<.05$.

AMS, acute mountain sickness; CO, cardiac output; DBP, diastolic blood pressure; HR, heart rate; IVC, inferior vena cava; LVOT, left ventricular outflow tract; $\mathrm{O}_{2}$ Sat, oxygen saturation; PLR, passive leg raise; SBP, systolic blood pressure; SV, stroke volume; VTI, velocity-time integral.

Gatterer et $\mathrm{al}^{32}$ demonstrate early fluid retention in individuals with acute AMS. Our results support the hypothesis that individuals who experience AMS symptoms fail to undergo significant bicarbonate diuresis and retain intravascular volume.

In our control group, mean LVOT VTI was increased by $18 \%$ after PLR maneuver, compared with an $11 \%$ increase in the AMS group $(P=.0088)$. Using prior literature supporting an increase of $12 \%$ or more being diagnostic of a fluid-responsive state, our data suggest that individuals without AMS can be described as having a lower circulatory volume status compared with individuals experiencing AMS, given the more robust increase in LVOT VTI. ${ }^{24,26}$ It should be noted that LVOT VTI measurements inherently have a wide range of individual variability, and therefore are likely more sensitive when serial individual measurements are performed. Based on our current LVOT VTI measurements, however, subjects with AMS have a statistically significantly larger circulating blood volume compared with 
Table 2. Acute mountain sickness score distribution based on Lake Louise scoring criteria

\begin{tabular}{lc}
\hline AMS score & Subject No. \\
\hline 0 & 21 \\
1 & 16 \\
2 & 14 \\
3 & 6 \\
4 & 4 \\
5 & 2 \\
6 & 0 \\
7 & 3 \\
8 & 1 \\
9 & 2 \\
\hline
\end{tabular}

AMS, acute mountain sickness.

asymptomatic individuals. There was no statistically significant difference between groups when evaluating IVC CI, and mean IVC CI was similar between two groups. Use of IVC CI has been reported for evaluation of hypovolemic volume status within the emergency department with mixed efficacy. An IVC collapse of greater than $50 \%$ is strongly suggestive of hypovolemic volume status. Unless the IVC is plethoric, an IVC CI between $10 \%$ and $50 \%$ is difficult to interpret. In our study, groups averaged $30 \%$ collapsibility, which makes their volume status difficult to discern. As noted previously, IVC CI is less sensitive than LVOT VTI in assessing volume status.

We performed a brief subgroup analysis of acetazolamide use in both AMS and control subjects to evaluate acetazolamide as a potential confounding variable. A statistically significant difference was noted between subjects with and without AMS in the group that did not use acetazolamide, with asymptomatic individuals having lower intravascular volume measured by \%Inc LVOT VTI $(18.41 \% \pm 13.7 \%$ control vs $7.75 \% \pm$ $17.92 \%$ AMS; $P=.006$ ). A similar trend was seen in both AMS and control subjects using acetazolamide, but it did not meet statistical significance. Interestingly, when comparing control groups who both did and did not use acetazolamide, \%Inc LVOT VTI values were similar, suggesting similar intravascular volume status and successful altitude-related diuresis. When comparing AMS groups, however, the group that took acetazolamide trended toward having a lower intravascular volume status than the one that did not, suggesting that the group that did take acetazolamide had at least a partially successful diuresis, as would be expected.

Several limitations to our study were encountered. Because of decreased patient presentation to Manang, Nepal, it was difficult to enroll the anticipated number of AMS subjects. Based on previous Himalayan Rescue
Association records, the Himalayan Rescue Association clinic was expecting to see approximately 60 to 100 patients with AMS for the fall season. However, during the fall season 2014, only 42 patients were seen in the clinic for AMS. Of the 42 cases seen during the 2.5 months of clinical practice, 29 were assessed for enrollment, and ultimately only 18 met the criteria for inclusion in the study, which likely introduced some selection bias. There was also a disproportionately increased use of acetazolamide in subjects with AMS as compared with healthy control subjects. However, given acetazolamide induces $\left[\mathrm{HCO}^{-}\right]$diuresis and thus depletes intravascular volume, if acetazolamide use rates were similar, one would expect an even larger difference in intravascular volume would have been detected between those with AMS and healthy control subjects. Although subgroup analysis of acetazolamide suggests a trend toward a higher circulating volume in individuals with AMS, statistical significance was not met. As our study was not longitudinal, it was not possible to measure input vs output during the subjects' ascent, nor were we able to classify hydration status. In addition, US measurements were taken by an individual practitioner, and LVOT VTI measurements could not be blinded because of technical limitations. Given LVOT VTI measurements have significant individual variability, and our study evaluated subjects at a single point, sequential measurements taken during ascent would make an excellent follow-up study.

\section{Conclusions}

Our study demonstrates that US assessment of intravascular volume status can be successfully deployed for use in wilderness settings. In the evaluation of subjects both with and without AMS who underwent ascent to $3519 \mathrm{~m}$ (11,545 feet) over 5 to 8 days, IVC CI measurement did not detect a significant difference in intravascular volume status between groups. Measurement of LVOT VTI before and after PLR, however, met statistical significance $(P=.0088)$, suggesting that individuals with AMS have a higher circulating blood volume than an altitude-matched control group. Our LVOT VTI data support the hypothesis of blunted bicarbonate diuresis in individuals who experience AMS. Given the wide range of subject variability, it is not clear that a single LVOT VTI measurement would be a useful clinical adjunct.

\section{Acknowledgments}

SonoSite, Inc provided ultrasound (M-Turbo) for research purposes. Equipment was returned upon completion of study. 


\section{References}

1. Sutton JR, Coates G, Houston CS, eds. The Lake Louise consensus on the definition and quantification of altitude illness. In: Hypoxia and Mountain Medicine. Burlington, VT: Queen City Printers; 1992.

2. Roach RC, Maes D, Sandoval D, et al. Exercise exacerbates acute mountain sickness at simulated high altitude. J Appl Physiol. 2000;88:581-585.

3. Hackett PH, Roach RC. High-altitude illness. $N$ Engl $J$ Med. 2001;345:107-114.

4. MacInnis MJ, Wang P, Koehle MS, Rupert JL. The genetics of altitude tolerance: the evidence for inherited susceptibility to acute mountain sickness. J Occup Environ Med. 2011;53:159-168.

5. Honigman B, Theis MK, Koziol-McLain J, et al. Acute mountain sickness in a general tourist population at moderate altitudes. Ann Intern Med. 1993;118:587-592.

6. Hackett PH, Rennie D, Levine HD. The incidence, importance, and prophylaxis of acute mountain sickness. Lancet. 1976;2:1149-1155.

7. Gallagher SA, Hackett PH. High-altitude illness. Emerg Med Clin North Am. 2004;22:329-355.

8. Vardy J, Vardy J, Judge K. Acute mountain sickness and ascent rates in trekkers above $2500 \mathrm{~m}$ in the Nepali Himalaya. Aviat Space Environ Med. 2006;77:742-744.

9. Hackett PH, Rennie D, Grover RF, Reeves JT. Acute mountain sickness and the edemas of high altitude: a common pathogenesis? Respir Physiol. 1981;46:383-390.

10. Gatterer H, Wille M, Faulhaber M, et al. Association between body water status and acute mountain sickness. Calbet JAL, ed. PLoS ONE. 2013;8:e73185.

11. Singh I, Khanna PK, Srivastava MC, Lal M, Roy SB, Subramanyam CS. Acute mountain sickness. N Engl J Med. 1969;280:175-184.

12. Richalet JP, Keromas A, Dersch B, et al. Physiological characteristics of high altitude climbers [Caratéristiques physiologiques des alpinistes de haute altitude]. Sci Sports. 1988;3:89-108.

13. Valli G, Bonardi D, Campigotto F, et al. Relationship between individual ventilatory response and acute renal water excretion at high altitude. Respir Physiol Neurobiol. 2008;162:103-108.

14. Imray C, Wright A, Subudhi A, Roach R. Acute mountain sickness: pathophysiology, prevention, and treatment. Prog Cardiovasc Dis. 2010;52:467-484.

15. Jabot J, Teboul JL, Richard C, Monnet X. Passive leg raising for predicting fluid responsiveness: importance of the postural change. Intensive Care Med. 2009;35:85-90.

16. Thiel SW, Kollef MH, Isakow W. Non-invasive stroke volume measurement and passive leg raising predict volume responsiveness in medical ICU patients: an observational cohort study. Crit Care. 2009;13:R111.

17. Kitakule MM, Mayo P. Use of ultrasound to assess fluid responsiveness in the intensive care unit. Open Crit Care Med J. 2010;3:33-37.
18. Fields JM, Lee PA, Jenq KY, Mark DG, Panebianco NL, Dean AJ. The interrater reliability of inferior vena cava ultrasound by bedside clinician sonographers in emergency department patients. Acad Emerg Med. 2011;18:98-101.

19. Marik PE, Monnet X, Teboul JL. Hemodynamic parameters to guide fluid therapy. Ann Intensive Care. 2011;1:1.

20. Haydar SA, Moore ET, Higgins GL, Irish CB, Owens WB, Strout TD. Effect of bedside ultrasonography on the certainty of physician clinical decisionmaking for septic patients in the emergency department. Ann Emerg Med. 2012;60(346-358):e4.

21. Muller L, Bobbia X, Toumi M, et al. Respiratory variations of inferior vena cava diameter to predict fluid responsiveness in spontaneously breathing patients with acute circulatory failure: need for a cautious use. Crit Care. 2012;16:R188.

22. Kalantari K, Chang JN, Ronco C, Rosner MH. Assessment of intravascular volume status and volume responsiveness in critically ill patients. Kidney Int. 2013;83:1017-1028.

23. Maizel J, Airapetian N, Lorne E, Tribouilloy C, Massy Z, Slama M. Diagnosis of central hypovolemia by using passive leg raising. Intensive Care Med. 2007;33:11331138.

24. Monnet X, Teboul JL. Passive leg raising. Intensive Care Med. 2008;34:659-663.

25. Monnet X, Teboul JL. Assessment of volume responsiveness during mechanical ventilation: recent advances. Crit Care. 2013; 17:217.

26. Lamia B, Ochagavia A, Monnet X, Chemla D, Richard C, Teboul JL. Echocardiographic prediction of volume responsiveness in critically ill patients with spontaneously breathing activity. Intensive Care Med. 2007;33:11251132.

27. Royse CF, Seah JL, Donelan L, Royse AG. Point of care ultrasound for basic haemodynamic assessment: novice compared with an expert operator. Anaesthesia. 2006;61: 849-855.

28. Baggish AL, Fulco CS, Muza S, et al. The impact of moderate-altitude staging on pulmonary arterial hemodynamics after ascent to high altitude. High Alt Med Biol. 2010;11:139-145.

29. Fagenholz PJ, Gutman JA, Murray AF, Noble VE, Thomas SH, Harris NS. Chest ultrasonography for the diagnosis and monitoring of high-altitude pulmonary edema. Chest. 2007;131:1013.

30. Otto C, Hamilton DR, Levine BD, et al. Into thin air: extreme ultrasound on Mt Everest. Wilderness Environ Med. 2009;20:283-289.

31. Wimalasena Y, Windsor J, Edsell M. Using Ultrasound lung comets in the diagnosis of high altitude pulmonary edema: fact or fiction? Wilderness Environ Med. 2013;24:159-164.

32. Loeppky JA, Icenogle MV, Maes D, Riboni K, HinghoferSzalkay H, Roach RC. Early fluid retention and severe acute mountain sickness. J Appl Physiol. 2004;98: 591-597. 